\title{
Prevalence and Antimicrobial Susceptibility Profile of Bacterial Isolates from Infected Caesarean Sites in Three Federal Capital Territory Hospitals, Abuja Nigeria
}

\author{
Egbe Friday Andrew ${ }^{1}$, Unam Nse Friday ${ }^{2}$, Egbe Kingsley Andrew ${ }^{3,}$, Lerum Nathaniel Isaiah $^{3}$, \\ Ekwom Edith Silas ${ }^{4}$, Unah Victor Unah ${ }^{3}$ \\ ${ }^{1}$ Department of Public Health, Institute of Federal Capital Territory Administration, Abuja, Nigeria \\ ${ }^{2}$ Department of Research, Federal Ministry of Health Institute, Abuja, Nigeria \\ ${ }^{3}$ Department of Microbiology, University of Nigeria, Nsukka, Nigeria \\ ${ }^{4}$ Department of Biological Science, Cross River University of Technology, Calabar, Nigeria
}

\section{Email address:}

fridandy@yahoo.co.uk (E. F. Andrew), unamnsefriday@yahoo.com (U. N. Friday), Kingegbe@yahoo.com (E. K. Andrew), natstarfish2010@gmail.com (L. N. Isaiah), egbe_edith@gmail.com (E. E. Silas), sirvicks84@gmail.com (U. V. Unah)

${ }^{*}$ Corresponding author

\section{To cite this article:}

Egbe Friday Andrew, Unam Nse Friday, Egbe Kingsley Andrew, Lerum Nathaniel Isaiah, Ekwom Edith Silas, Unah Victor Unah. Prevalence and Antimicrobial Susceptibility Profile of Bacterial Isolates from Infected Caesarean Sites in Three Federal Capital Territory Hospitals, Abuja Nigeria. American Journal of Biomedical and Life Sciences. Vol. 6, No. 4, 2018, pp. 90-95. doi: 10.11648/j.ajbls.20180604.15

Received: August 10,2018; Accepted: September 21, 2018; Published: October 25, 2018

\begin{abstract}
In Nigeria, delivery by caesarean section which was once a dreaded event due in part to possible complications, fear and cost implications has over the past few years gain acceptance especially among the urban dwellers, as more women and doctors opt for caesarean delivery, resulting to increase in incidence of caesarean site infections. A total of one hundred and ninety four (194) caesarean sites of women who have undergone caesarean section delivery in three federal capital territory hospitals with signs of infection were screened for bacterial infection between September, 2017 and July 2018 . Specimens were collected using sterile cotton swab and processed using standard operative procedures in appropriate culture media and susceptibility test was done using Kirby-Bauer disc diffusion technique. The result showed that forty six (46) out of the total (194) were found to be infected. This represented a $23.71 \%$ infection rate. The infection was polymicrobial in nature with various bacterial species such as; Escherichia coli, Enterobacter spp, Staphylococcus aureus, Staphylococcus epidermidis, Pseudomonas aeruginosa, Klebsiella spp and Proteus spp, isolated. The single most commonly infecting organism was found to be Escherichia coli which had been isolated from 13(28.26\%) samples, followed by Staphylococcus aureus $10(21.74 \%)$, Staphylococcus epidermidis 8(17.39\%), Enterobacter spp 6(13.04), Pseudomonas aeruginosa 4(8.70\%), Klebsiella spp $3(6.52 \%)$, and Proteus spp 2(4.35\%) respectively. One hundred percent (100\%) resistance to tetracycline and amoxicillin was recorded mostly from gram negative organisms while ciprofloxacin, gentamycin, cefalexin and cefuroxime exhibited significant antibacterial activity against the isolated pathogens and therefore recommended for consideration in cases of caesarean infection.
\end{abstract}

Keywords: Cesarean Sites, Staphylococcus Aureus, Antibiotics, Antimicrobial Susceptibility Profile

\section{Introduction}

Infection in a wound is a manifestation of disturbed hostbacteria equilibrium in favor of bacteria [1]. A wound infection is defined by the US Centre for Disease Control and Prevention (CDC) as surgical site infection [2]. Surgical site infections (SSIs) are defined as infections that occur during one month after a surgical operation or one year after implant surgery and affecting either the injury site or near surgical injuries [3]. Internationally, surgical site infections (SSI) make up a large percentage of hospital-acquired infections [4]. Surgical site skin infections (SSIs) are preventable 
complication of delivery via caesarean section [5]. The Centers for Disease Control and Prevention (CDC) estimates that SSIs complicate approximately $5 \%$ of the nearly 30 million surgeries performed each year [6]. Surgical site infections (SSIs) results in a significant morbidity and mortality throughout the world [1], and are one of the most common hospital-acquired infections, causing higher patient mortality and significantly longer length of stay [7]. Due to heterogeneous nature of these surgical infections, studies of the epidemiology of SSIs are very difficult [8]. The incidence differs widely between surgical procedures, hospitals, patients and surgeons [9]. Despite the technical advancement in infection control and surgical practices, these infections still continue to be a major problem, even in hospitals with advanced modern facilities [8]. Beyond infection and surgical wounds, of increasing concern is the problem of antibiotic resistance. As the prevalence of antibiotic resistant strains have emerged in many bacterial species that cause disease on humans, thereby threatening the future of most commonly used antibiotics. With the rising number of caesarean deliveries and increasing cases of post caesarean infections especially in rural areas, it becomes necessary to identify the agents responsible for caesarean sites infection, as well as to determine the extent of their susceptibility to commonly used antibiotics, in order to make relevant health recommendations aimed at preventing, controlling or managing caesarean site infections as our objectives necessitated this research.

\section{Materials and Methods}

\subsection{Sample}

A total of one hundred and ninety-four (194) samples were obtained from patients in three hospitals; Nyanya General Hospital, Kubwa General Hospital and Federal Staff Hospital within Federal Capital Territory Abuja, between September 2017 and July 2018. Patients who had undergone caesarean operation for delivery within 30 days with the wounds showing signs of infection were enrolled into the study. Patients consent and approval was obtained before samples were obtained for the study. A single sterile swab stick (Evepon sterile swab stick by Evepon industries Ltd) was used by swabbing the pus and drainage from the caesarean site. The swab specimens were transported immediately to the Microbiology laboratory units of the hospitals for processing.

\subsection{Culture and Identification of Bacterial Species}

Samples were inoculated into Nutrient agar (NA), Blood agar (BA), Chocolate agar (CHO) and MacConkey agar (MCA). Primary cultures were sub cultured using the streaking techniques and to obtain pure cultures. Cultures were incubated for 24 hours at $37^{\circ} \mathrm{C}$ for differential agar (MCA), while enriched cultures (BA, CHO) were incubated under candle jar at $37^{\circ} \mathrm{C}$ for 24 hours. Isolated colonies were identified using gram stain and standard biochemical procedures.

\subsection{Antimicrobial Susceptibility Testing}

This was performed on isolated pathogenic organisms using commonly used antibiotics. A total of nine (9) different commonly used antibiotics were used. The antibiotics were already prepared into antibiotic disc in micrograms ( $\mathrm{mcg}$ ) by Himedia Laboratories Ltd, India. The antibiotics are Cefuroxime (30 mcg), Cefalexin $(30 \mathrm{mcg})$, Ciprofloxacin (5mcg), Amoxicillin (10 mcg), Gentamycin (10 mcg), Erythromycin (15mcg), Augmentin (30mcg), Cotrimoxazole $(25 \mathrm{mcg})$ and Tetracycline $(30 \mathrm{mcg})$. Zones of inhibition were measured after 24 hours incubation at $37^{\circ} \mathrm{C}$ on Mueller Hinton agar using a meter rule in millimeters. Interpretation as sensitive or resistant is based on approved standards of the antibiotics in microgram ( $\mathrm{mcg}$ ) concentrations by national committee on clinical laboratory standards. Modified KirbyBaur disc diffusion technique was used.

\subsection{Data Analysis}

Data generated were collated, entered into IBM SPSS version 22 and statistical significance evaluated using the Ttest method at $95 \%$ Confidence limit. The results of the analysis are presented in simple percentages, tables and graph for easy comprehension.

\section{Results}

A total of one hundred and ninety four (194) caesarean sites were screened for bacterial infection. The result showed that forty six (46) out of the total (194) were found to be infected. This represented a $23.71 \%$ infection rate. The infection was polymicrobial in nature, with various bacterial species such as; Escherichia coli, Staphylococcus aureus, Staphylococcus epidermidis, Enterobacter spp, Pseudomonas spp, Klebsiella spp and Proteus spp.

Table 1. Prevalence of Bacterial Species from Infected Caesarean Sites.

\begin{tabular}{lll}
\hline Bacterial species & Number of samples & Prevalence (\%) \\
\hline Escherichia coli & 46 & $13(28.26)$ \\
Staphylococcus aureus & 46 & $10(21.74)$ \\
Staphylococcus epidermidis & 46 & $8(17.39)$ \\
Enterobacter spp & 46 & $6(13.04)$ \\
Pseudomonas aeruginosa & 46 & $4(8.70)$ \\
Klebsiella spp & 46 & $3(6.52)$ \\
Proteus spp & 46 & $2(4.35)$ \\
\hline
\end{tabular}

Numbers in Brackets are in percentages. $46<0.05$

From the results, the single most commonly infecting organism was found to be Escherichia coli which had been isolated from 13(28.26\%) samples. Staphylococcus aureus caused 10(21.74\%) infections, while 8(17.39), 6(13.04\%), $4(8.70 \%), 3(6.52 \%)$ and $2(4.35 \%)$ were caused by Staphylococcus epidermidis, Enterobacter spp, Pseudomonas aeruginosa, Klebsiella spp, and Proteus spp respectively as shown in Table1 and Figure 1. Statistically there was a significant difference in the occurrence of the various bacterial species. 


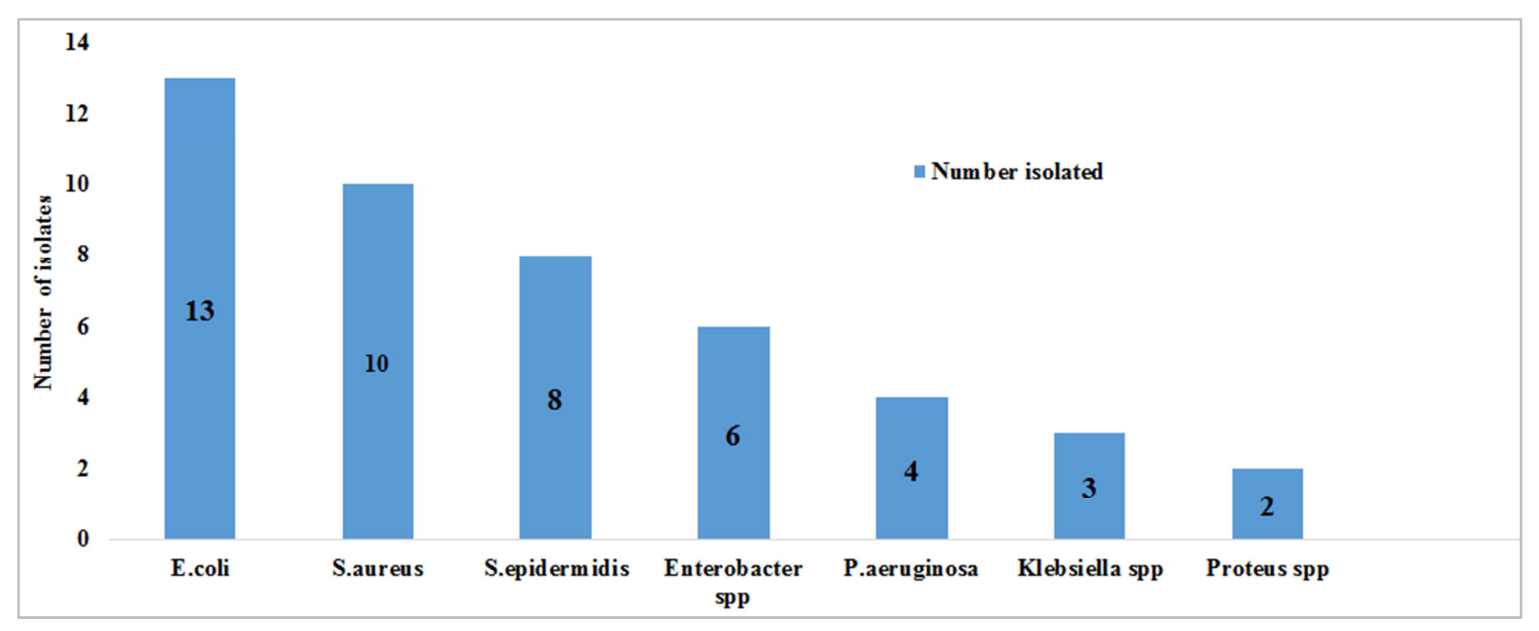

Figure 1. Showing bacteria isolated and the frequency of isolation.

Table 2 shows the result of the antimicrobial susceptibility profile of nine (9) different, commonly used antibiotics against three (3) gram positive organisms isolated from infected caesarean sites in selected hospitals in Abuja, as presented in table 2. From the results, S. aureus was most susceptible to ciprofloxacin and cefuroxime with $83.3 \%$ susceptibility, followed by cefalexin and erythromycin $(66.7 \%)$, augmentin and gentamycin $(50 \%)$ and amoxicillin (33.3\%) while there was $83.3 \%$ resistance to tetracycline. In S. epidermidis, ciprofloxacin and Cefuroxime both recorded 75.5\% susceptibility, followed by Amoxicillin (66.7\%), gentamycin, erythromycin, cefalexin and augmentin all recorded $62.5 \%$ susceptibility. Tetracycline and cotrimoxazole were the most resisted drugs by S. epidermidis with $66.7 \%$ and $62.5 \%$ resistance respectively. Also, based on the results, Enterobacter spp, were most susceptible to cefalexin and ciprofloxacin with $80 \%$ susceptibility, followed by cefuroxime (70\%), augmentin, amoxicillin and gentamycin (60\%) and erythromycin (50\%). There was $80 \%$ and $70 \%$ resistance to tetracycline and cotrimoxazole respectively by Enterobacter spp.

Table 2. Antibiotic Susceptibility Pattern of Staphylococcus and Enterobacter spp Isolated from Infected Caesarean Sites.

\begin{tabular}{llll}
\hline Antibiotics & S. aureus (N=6) NS NR & S. epidermidis (N=8) NS NR & Enterobacter spp (N=10) NS NR \\
\hline Amoxicillin & $2(33.3) 4(66.7)$ & $4(66.7) 2(33.3)$ & $6(60.0) 4(40.0)$ \\
Augmentin & $3(50.0) 3(50.0)$ & $5(62.5) 3(37.5)$ & $6(60.0) 4(40.0)$ \\
Cefalexin & $4(66.7) 2(33.3)$ & $5(62.5) 3(37.5)$ & $8(80.0) 2(20.0)$ \\
Cefuroxime & $5(83.3) 1(16.7)$ & $6(75.0) 2(15.0)$ & $7(70.0) 3(30.0)$ \\
Ciprofloxacin & $5(83.3) 1(16.7)$ & $6(75.0) 2(15.0)$ & $8(80.0) 2(20.0)$ \\
Cotrimoxazole & $2(33.3) 4(66.7)$ & $3(37.5) 5(62.5)$ & $3(30.0) 7(70.0)$ \\
Erythromycin & $4(66.7) 2(33.3)$ & $5(62.5) 3(37.5)$ & $5(50.0) 5(50.0)$ \\
Gentamycin & $4(50.0) 4(50.0)$ & $5(62.5) 3(37.5)$ & $6(60.0) 4(40.0)$ \\
Tetracycline & $1(16.7) 5(83.3)$ & $2(33.3) 6(66.7)$ & $2(20.0) 8(80.0)$ \\
\hline
\end{tabular}

NS-=Number of Sensitive Organisms. NR=Number of Resistant Organisms. N= Number of Isolates tested. The Numbers in bracket are in percentages.

Table 3 shows the result of the antimicrobial susceptibility pattern of nine (9) different commonly used antibiotics to four (4) gram negative organisms isolated from caesarean sites in selected FCTA hospitals in Abuja, as presented in the Table 3.

From the results, E. coli was most susceptible to ciprofloxacin and Gentamycin with $69.2 \%$ susceptibility, followed by erythromycin $61.5 \%$ and cefuroxime $53.4 \%$. There was a $92.3 \%$ resistance to tetracycline, followed by cotrimoxazole $(84.6 \%)$, augmentin $(76.9 \%)$ and amoxicillin $(53.4 \%)$ respectively in a decreasing order of resistance. In Klebsiella spp, the most effective drugs in inhibiting its growth were amoxicillin, cefalexin, cefuroxime, ciprofloxacin and erythromyxin all with 50\% susceptibility rating. While there was $100 \%$ resistance to tetracycline and cotrimoxazole, followed by augmentin and gentamycin
(75\%). Klebsiella spp had $50 \%$ susceptibility to ciprofloxacin, augmentin, cefuroxime, erythromycin and Gentamycin respectively. While there was $100 \%$ resistance to tetracycline, followed by gentamycin and augmentin with $75 \%$ resistance. Ciprofloxacin was the most effective drug in inhibiting the growth of Proteus spp with $100 \%$ susceptibility, followed by augmentin, cefuroxime, erythromycin and gentamycin all recorded $50 \%$ susceptibility. However, there was $100 \%$ resistance to amoxicillin, cotrimoxazole and tetracycline. Gentamycin was the most effective drug in inhibiting the growth of Pseudomonas spp with $66.3 \%$ susceptibility. However as shown in the table (Table 3), there was $100 \%$ resistance to tetracycline, cotrimoxazole, cefuroxine, cefalexin and amoxicillin. 
Table 3. Antibiotic Susceptibility Pattern of Different Gram Negative Bacterial Isolates from Infected Caesarean sites.

\begin{tabular}{|c|c|c|c|c|}
\hline Antibiotics & E.coli $(N=13)$ NS NR & Klebsiella spp $(\mathrm{N}=4)$ NS NR & Proteus spp $(\mathrm{N}=2)$ NS NR & $P$. aeruginosa $(\mathrm{N}=3) \mathrm{NS} N \mathrm{NR}$ \\
\hline Amoxicillin & $6(46.6) 7(53.4)$ & $2(50.0) 2(50.0)$ & $0(0.00) 2(100.0)$ & $0(0.00) 3(100.0)$ \\
\hline Augmentin & $3(23.1) 10(76.9)$ & $1(25.0) 3(75.0)$ & $1(50.0) 1(50.0)$ & $1(33.3) 2(66.7)$ \\
\hline Cefalexin & $6(46.6) 7(53.4)$ & $2(50.0) 2(50.0)$ & $0(0.00) 2(100.0)$ & $0(0.00) 3(100.0)$ \\
\hline Cefuroxime & $7(53.4) 6(46.6)$ & $2(50.02(50.0)$ & $1(50.0) 1(50.0)$ & $0(0.00) 3(100.0)$ \\
\hline Ciprofloxacin & $9(69.2) 4(30.8)$ & $2(50.0) 2(50.0)$ & $2(100.0) 0(0.00)$ & $1(33.3) 2(66.7)$ \\
\hline Cotrimoxazole & $2(15.4) 11(84.6)$ & $0(0.00) 4(100.0)$ & $0(0.00) 2(100.0)$ & $0(0.00) 3(100.0)$ \\
\hline Erythromycin & $8(61.5) 5(38.5)$ & $2(50.0) 2(50.0)$ & $1(50.0) 1(50.0)$ & $1(33.3) 2(66.7)$ \\
\hline Tetracycline & $1(7.7) 12(92.3)$ & $0(0.00) 4(100.0)$ & $0(0.00) 2(100.0)$ & $0(0.00) 3(100)$ \\
\hline
\end{tabular}

NS-=Number of Sensitive Organisms. NR=Number of Resistant Organisms. N= Number of Isolates tested.

The Numbers in bracket are in percentages.

\section{Discussion}

Surgical site infection (SSI) is the second most common infectious complication after urinary tract infection following a delivery by caesarean section [10]. Caesarean section (CS) rates have increased globally during the past three decades [11]. In Nigeria, delivery by caesarean section which was once a dreaded event due in part to possible complications and fear has over the past few years gain acceptance, especially among the urban dwellers as more women and doctors opt for caesarean delivery. This increase can be linked to inability to deliver normally through the vagina, unwillingness of women to go through the pain and rigors of child birth and shorter delivery time has made caesarean delivery very common. This study was undertaken to identify the prevalence and aetiologic agents responsible for caesarean site infection and to determine the susceptibility of the isolated infecting agents to common antibiotics in selected hospitals in FCT Abuja. From our findings, based on the result obtained, there was a $23.71 \%$ infection rate of caesarean sites. The global occurrence of an SSI following a Caesarean section (CS) varies remarkably. In New Zealand, a $5 \%$ infection rate has been reported [12], while a $10.9 \%$ rate was reported in Tanzania [10]. The development of post CS infection depends on a complex interplay of many factors including wound class, immune status, maternal age, hypertensive disorders, ASA classification, numbers of vaginal examinations, the virulence of the microorganisms, maternal weight, surgical techniques and premature rupture of membrane [13], [14]. Pathogens that infect CS surgical wounds can be part of the patient's normal flora (endogenous source), originate from the skin, vaginal and peritoneal cavities, or can be acquired from the hospital environment, other infected patients, and surgeons (exogenous source) [15], [16]. Among the etiological agents isolated, Escherichia coli was the most common organism 13(28.26\%). Other isolates include, Staphylococcus aureus 10(21.74\%), Staphylococcus epidermidis 8(17.39\%), Enterobacter spp $6(13.04 \%)$, Pseudomonas aeruginosa $4(8.80 \%)$, Klebsiella spp 3(6.52\%) and Proteus spp 2(4.35\%) respectively. Mpogoro et al. [10], also isolated Staphylococcus aureus, Klebsiella spp, Pseudomonas spp, Proteus spp and Enterobacter spp however they found Staphylococcus aureus as the predominant isolate in contrast to our findings of
Escherichia coli being the predominant aetiologic agent, Zuarez-Easton et al. [17], also stated in their review that Staphylococcus aureus is frequently the most isolated pathogen in caesarean site infection.

Successful management of patients with bacterial infection depends on early identification of bacterial pathogens and selection of an effective antibiotic against the organism [18]. In this study, the isolates were tested against nine (9) commonly used antibiotics. It is evident from our results, that the most effective antibiotics for the treatment of infections caused by Staphylococcus aureus is ciprofloxacin and cefuroxime both with $83.3 \%$ susceptibility while the least antibiotics of choice against Staphylococcus aureus was tetracycline with $83.3 \%$ resistance rate. Erythromycin was also active against $\mathrm{S}$. aureus with $66.7 \%$ susceptibility. In their study to determine bacterial pathogens and drug susceptibility profile of pathogens isolated from surgical site infections at St. Paul Specialized Hospital Millennium Medical College and Yekatit 12 Referral Hospital Medical College, Addis Ababa-Ethiopia, Walelign et al. [18], recorded $78.9 \%$ erythromycin susceptibility against S. aureus. However, their findings of $78.9 \%$ tetracycline susceptibility varies remarkably with our result of $83.3 \%$ tetracycline resistance. Enterobacter spp also showed a very high resistance to tetracycline (80\%) and erythromycin (70\%). Our findings $50 \%$ gentamycin and $66.7 \%$ S. aureus resistance to amoxicillin and cotrimoxazole is vindicated by another study on SSI in Ethiopia by Kahsay et al. [19], who recorded $80 \%$ amoxicillin, $95.9 \%$ erythromycin and $97.2 \%$ cotrimoxazole resistance by S. aureus. However, in contrast to our findings, they recorded $50.7 \%$ susceptibility to tetracycline by $\mathrm{S}$. aureus in their report on antimicrobial susceptibility pattern of Staphylococcus aureus in patients with surgical site infection at Debre Markos Referral Hospital, Amhara Region, Ethiopia. Also, in contrast to our findings of $83.3 \%$ Staphylococcus aureus susceptibility to ciprofloxacin, Chhetry et al. [20], in Nepal reported 86.2\% Staphylococcus aureus resistance of ciprofloxacin.

Gram negative organisms isolated in this study were found to exhibit a high level of resistance to these commonly used antibiotics. From the results, there was a $92.3 \%$ tetracycline, $84.6 \%$ cotrimoxazole, $76.9 \%$ augmentin and $53.4 \%$ amoxicillin and cefalexin resistance by E. coli. There was $100 \%$ resistance to tetracycline and cotrimoxazole as well as 
$75 \%$ augmentin resistance by Klebsiella spp. In Proteus spp, there was $100 \%$ resistance to tetracycline, cotrimoxazole, cefalexin and amoxicillin while in Pseudomonas aeruginosa we recorded also like other gram negative organisms a $100 \%$ resistance to tetracycline, cotrimoxazole, cefuroxime, cefalexin and amoxicillin. Pseudomonas aeruginosa was however susceptible to gentamycin treatment with $66.7 \%$ susceptibility. In consonance with our findings, Walelign et al. [18], in a similar study in Ethiopia also reported $100 \%$ tetracycline resistance by Proteus spp, 83.3\% tetracycline resistance by E. coli. In Palestine, Ghaleb et al. [8], in their work on surgical site infections also reported a $100 \%$ resistance to tetracycline by Klebsiella spp as well as $58.5 \%$ ciprofloxacin resistance by E. coli. However their findings of $100 \%$ resistance of gentamycin by Klebsiella spp varies with our report of $75 \%$ resistance. Also in a justification of our findings of very high resistance to commonly used antibiotics by gram negative organisms, Chhetry et al. [20], in Nepal reported $80 \%$ ciprofloxacin and erythromycin resistance and $100 \%$ cotrimoxazole resistance by E. coli while a $100 \%$ ciprofloxacin and erythromycin resistance by Klebsiella spp was also recorded.

\section{Conclusions}

The study reveals a high rate of caesarean site infection within the federal capital territory, Abuja and its environs. The nature of the infection of the caesarean sites was polymicrobial, with the isolated pathogens exhibiting a high level of resistance to commonly used antibiotics. Therefore more efforts should be made to educate pregnant women during anti-natal care program to also take responsibility for care and prevention of caesarean sites or wounds. The practice of aseptic techniques during and after surgery should continue to be emphasized while over reliance and use of antibiotics deemphasized. And as such, rational use of antibiotics and continuing surveillance of bacterial susceptibility is necessary to curb the continuous rise and spread of resistant bacterial pathogens. Generally, gentamycin, ciprofloxacin, cefuroxime and cephalexin should be considered in preference to tetracycline and amoxicillin in a case of caesarean site infection.

\section{Acknowledgements}

The authors acknowledge the support and assistance provided by the members of the laboratory unit and obstetrics and Gynecology department of the hospitals.

\section{References}

[1] A. Qaisar, N. Akhtar, R. W. Akhtar and W. latif, "Antimicrobial susceptibilty profile of bacterial pathogens in surgical site infections at a tertiary care hospital in Rawalpindi,” J. Inf. Mol. Biol, vol. 3, pp. 57-61, 2015.

[2] S. Madhavi, and S. S. Parveen, "Bacteriological Profile and
Antimicrobial Sensitivity of Wound Infection," Int. J. Curr. Microbiol. App. Sci, vol. 4, pp.248-254, 2015.

[3] T. C. Horan, R. P. Gaynes, W. J. Martone, W. R. Jarvis, T. G. Emori, "CDC definitions of noscomial surgical site infections: a modification of CDC definitions of surgical wound infections," Infect Control Hosp Epidemiol, vol.13, pp. 606608, 1992.

[4] T. Yoshio, K. Shinya, M. Hiroshige, S. Junko, W. Akira, K. Hiroshi, I. Satoshi, K. Mitsuo, H. Hideaki, S. Yoshinobu, K. Yuko, M. Toru, A. Yoshiyasu, K. Masafumi, I. Keiichiro, M. Akihisa, H. Kazuo, H. Yasushi, O. Koji, S. Koji, H. Takuo, M. Koshi, M. Yoichi, K. Minako, K. Shoji, U. Kazuhisa, S. Junzo, K. Ryohei, O. Hiroki, A. Shinji, O. Masaaki, W. Katsunori, O. Kohji, Y. Katsunori, "Antimicrobial susceptibility of pathogens isolated from surgical site infections in Japan: Comparison of data from nationwide surveillance studies conducted in 2010 and $2014-2015$," J Infect Chemother, vol. 23, pp. 339-348, 2017.

[5] K. Henman, C. L. Gordon, T. Gardiner, J. Thorn, B. Spain, J. Davies, R. Baird, "Surgical site infections following caesarean section at Royal Darwin Hospital, Northern Territory," Healthcare Infection, vol. 17, pp. 47-51, 2012.

[6] J. A. Weigelt, B. A. Lipsky, Y. P. Tabak, K. G. Derby, M. Kim, and V. Gupta, "Surgical site infections: causative pathogens and associated outcomes," Am. J. Infect. Control, vol. 38, pp. 112- 120, 2010.

[7] I. K. Afifi, E. A. Labah, and K. M. Ayad, "Surgical site infections after elective general surgery in Tanta University Hospital: Rate, risk factors and microbiological profile," Egyptian J. Med. Microbiol, vol. 18, pp. 61-71, 2009.

[8] A. Ghaleb, A. H. Nael, S. Ibrahim, S. Dalia, A. Shorouq, O. Shorouq, A. A. Zeinab, and B. Haneen, "Detection of bacterial pathogens in surgical site infections and their antibiotic sensitivity profile," Int J Med res Health Sci, vol. 5, pp. 75-82, 2016.

[9] R. L. Nichols, "Preventing surgical site infections: a surgeon's perspective" Emerging Infectious Diseases, vol. 7, pp. 220224, 2001.

[10] F. J. Mpogoro, S. E Mshana, M. M. Mirambo, B. R. Kidenya, B. Gumodoka, and C. Imirzalioglu "Incidence and predictors of surgical site infections following caesarean sections at Bugando Medical Centre, Mwanza, Tanzania" Antimicrobial Resistance and Infection Control vol. 3 (25), pp. 1-10, 2014.

[11] A. P. Betrán, M. Merialdi, J. A. Lauer, W. Bing - Shun, J. Thomas, P. Van Look, M. Wagner, "Rates of caesarean section: analysis of global, regional and national estimates," . Paediatr Perinat Epidemiol, vol. 21, pp. 98-113, 2007.

[12] M. Ghuman, D. Rohlandt, G. Joshy, and R. Lawrenson, "Postcaesarean section surgical site infection: rate and risk factors," NZMJ, Vol. 124 No 1339, pp. 32-36, 2011.

[13] M. A. Olsen, A. M. Butler, D. M. Willers, P. Devkota, G. A. Gross, V. J. Fraser, "Risk factors for surgical site infection after low transverse cesarean section," Risk, vol. 29, pp. 477484, 2008.

[14] S. P. Gong, H. X. Guo, L. Chen, Y. H. Yu, "Morbidity and risk factors for surgical site Infection following cesarean section in Guangdong Province, China," J Obstet Gynaecol Res, vol. 38, pp. 509-515, 2012. 
[15] A. Giacometti, O. Cirioni, A. Schimizzi, M. Del Prete, F. Barchiesi, M. D'errico, E. Petrelli, G. Scalise, "Epidemiology and microbiology of surgical wound infections," J Clin Microbiol, vol. 38, pp. 918-922, 2000.

[16] Anguzu J, Olila D: Drug sensitivity patterns of bacterial isolates from septic post-operative wounds in a regional referral hospital in Uganda. Afr Health Sci 2007, 7(3):148154.

[17] S. Zuarez-Easton, N, Zafran, G, Garmi, R, Salim, "Postcesarean wound infection: prevalence, impact, prevention, and management challenges" International Journal of Women's Health, vol. 9, pp. 81-88, 2017.

[18] D. Walelign, M. Gebru, F. Surafael, M. Amete, H. Mulu, and A. Engida, "Pattern of Bacterial Pathogens and Their Susceptibility Isolated from Surgical Site Infections at
Selected Referral Hospitals, Addis Ababa, Ethiopia," Int J Microbiol, vol. 2016, pp. 1-10, 2016. Retrieved from: http://dx.doi.org/10.1155/2016/2418902.

[19] A. Kahsay, A. Mihret, T. Abebe, and T. Andualem, "Isolation and antimicrobial susceptibility pattern of Staphylococcus aureus in patients with surgical site infection at Debre Markos Referral Hospital, Amhara Region, Ethiopia," Archives of Public Health, vol. 72, pp. 1-7, 2014. Retrieved from: https://archpublichealth.biomedcentral.com/articles/10.1186/2 049-3258-72-16.

[20] M. Chhetry, S. Subedi, S. Ghimire, S. Lamichanne, B. Banerjee, G. K. Singh, "Antibiotic sensitivity in post cesarean surgical site infection at a tertiary care centre in Eastern Nepal," Journal of Lumbini Medical College, vol. 4(2):55-59, 2016. 